\title{
Real-time measurements of tonal audibility by using multifunctional measuring system
}

\author{
Akiko Sugahara $^{1, *}$, Kohei Okubo ${ }^{2}$, Miki Yonemura ${ }^{3},{\text { Hyojin } \text { Lee }^{3} \text {, Shinichi Sakamoto }}^{3}$, \\ Yuichi Yonemoto ${ }^{4}$ and Toshiya Ohshima ${ }^{5}$ \\ ${ }^{1}$ Faculty of Architecture, Kindai University, 3-4-1 Higashiosaka, 577-8502 Japan \\ ${ }^{2}$ Department of Architecture, Graduate School of Engineering, The University of Tokyo, \\ 4-6-1 Komaba, Meguro-ku, Tokyo, 153-8505 Japan \\ ${ }^{3}$ Institute of Industrial Science, The University of Tokyo, 4-6-1 Komaba, Meguro-ku, Tokyo, 153-8505 Japan \\ ${ }^{4}$ RION Co., Ltd., 3-20-41 Higashimotomachi, Kokubunji, 185-8533 Japan \\ ${ }^{5}$ Aviation Environment Research Center, Organization of Airport Facilitation, \\ 1-3-1 Shibakoen, Minato-ku, Tokyo, 105-0011 Japan
}

(Received 5 January 2021, Accepted for publication 8 February 2021)

Keywords: Development of measuring system, Tonal audibility, Real-time measurement, Multifunctional measuring system

\section{Introduction}

Tonal components in noise, e.g., generated by wind turbines, may increase annoyance and cause complaints due to its characteristic sound, even with low level. The tonal audibility (hereinafter, TA), which is the evaluation index of the tonal components audibility, is provided by some international standard such as ISO 1996-2:2017 [1] (the calculation procedure is shown in ISO/PAS 20065:2016 [2]) and IEC 61400-11:2012 [3]. Their calculation procedures are generally the same with minor differences. According to Fletcher's theory, the narrow frequency band surrounding the tone in the critical band contributes the masking effect on the tone [4,5]. TA is the value calculated from the energy ratio of the tonal component to such masking noise added with the masking index determined in repeated tests with normal hearing subjects. ISO PAS 20065:2016 supplies a sample program to compute TA.

In some countries, tonal penalty is added to the noise indices (A-weighted sound pressure level, 1/3 octave band level, and so on) based on the evaluation index of audibility in the guideline for wind turbine noise [6]. There have been noise complaints in Japan with the increase in wind power facilities. Although the guideline published by the Ministry of the Environment provide guidance on A-weighted sound pressure level, there exists no clear criteria for pure tone components. Some Japanese researchers have been doing research on the tonal noise $[7,8]$ but are still in the study stage with a small amount of data. For the above circumstances, a simple and highly versatile measuring system based on the sample programs of the standards or specifications is desired to develop and maintain enough data. In this study, a TA measuring application working with a tablet device with AD conversion and FFT function (RIONOTE SA-A1, RION) for the trial. This application enables easy real-time field measurement.

*e-mail: sugahara@arch.kindai.ac.jp

[doi:10.1250/ast.42.202]

\section{Calculation algorithm}

Figure 1 shows the flowchart of the calculation algorithm of the prototype software complying with ISO/PAS 20065. The average A-weighted power spectrum is first calculated for more than three seconds. The critical band about the tone frequency $f_{\mathrm{T}}$ is given by:

$$
\begin{aligned}
\Delta f_{\mathrm{c}} & =25.0+75.0\left[1.0+1.4\left(\frac{f_{\mathrm{T}}}{1000}\right)^{2}\right]^{0.69} \\
f_{\mathrm{T}} & =\sqrt{f_{1} \cdot f_{2}} \\
f_{1} & =-\frac{\Delta f_{\mathrm{c}}}{2}+\frac{\sqrt{\left(\Delta f_{\mathrm{c}}\right)^{2}+4 f_{\mathrm{T}}^{2}}}{2} \\
f_{2} & =f_{1}+\Delta f_{\mathrm{c}}
\end{aligned}
$$

where $\Delta f_{\mathrm{c}}$ is the critical band width, and $f_{1}$ and $f_{2}$ are the corner frequencies of the band. The mean narrow-band level and the critical band level are then obtained. The mean narrow-band level $L_{\mathrm{S}}$ is the energy mean value in the critical band that does not exceed this value by more than $6 \mathrm{~dB}$. This is derived in the iteration procedure. The critical band level $L_{\mathrm{G}}$, which is the level of the band other than the tonal components, is calculated from $L_{\mathrm{S}}$ by the following equation.

$$
L_{\mathrm{G}}=L_{\mathrm{S}}+\left[10 \log _{10} \frac{\Delta f_{\mathrm{c}}}{\Delta f}\right]
$$

where $\Delta f$ is the frequency resolution. The frequency component is judged to be the tone and the tone level is $L_{T}$ if it satisfies some specified conditions, such as the level difference from the mean narrow-band level of the critical band $L_{\mathrm{S}}$, the narrowness of the tone bandwidth, and the edge steepness. The tonal audibility $\Delta L$ is calculated using the level difference of the tonal and the critical band level, taking into account the masking index $a_{\mathrm{V}}$.

$$
a_{\mathrm{V}}=-2-\log _{10}\left[1+\left(\frac{f}{502}\right)^{2.5}\right]
$$

Finally, the maximum $\Delta L$ in the entire spectrum is presented as TA. 


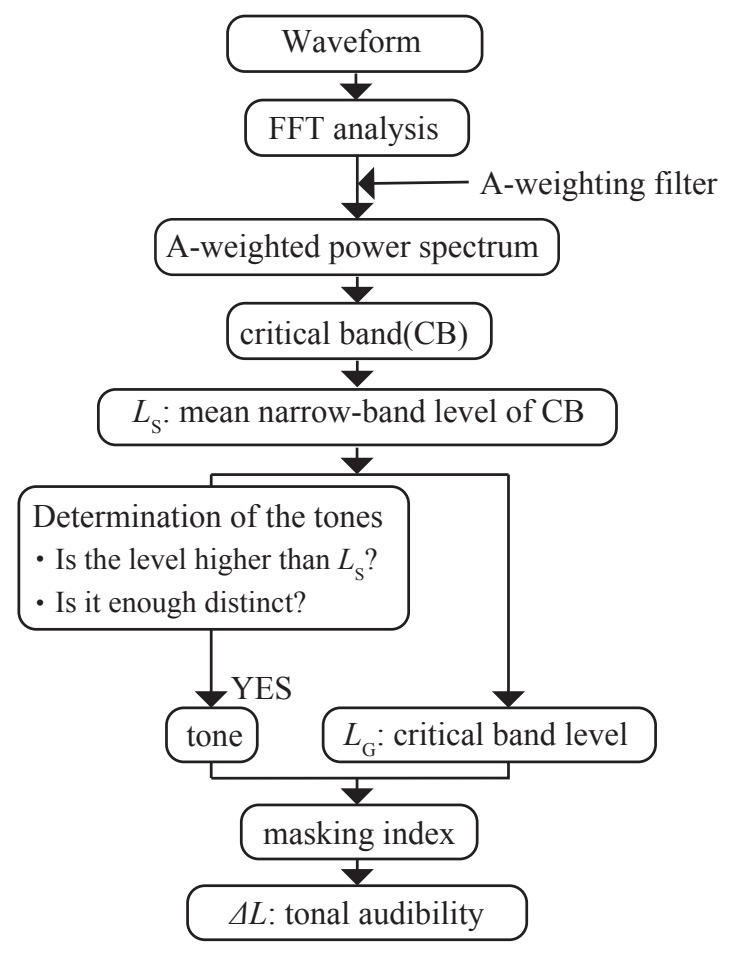

Fig. 1 TA calculating procedure of ISO/PAS 20065.

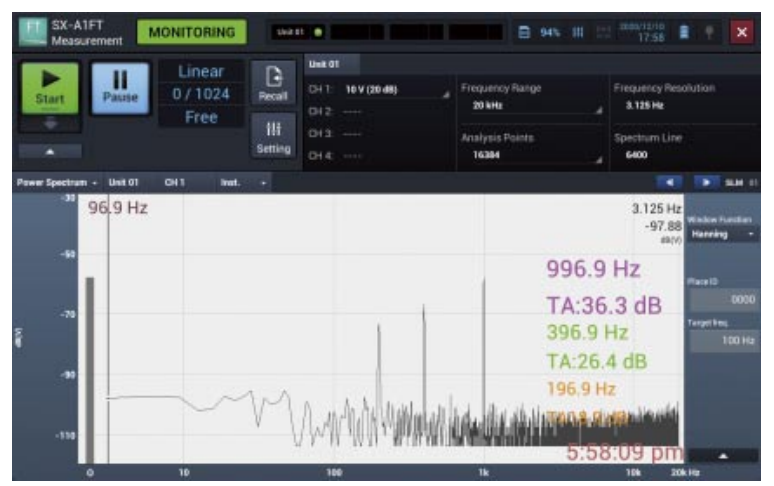

Fig. 2 Screen capture.

The prototype software works with a measuring device with $\mathrm{AD}$ conversion and FFT function connected to the microphones and sound level meters. It obtains the Aweighted power spectrum for three seconds using the FFT function of the device. It then calculates TA according to the procedure mentioned above. In the ISO/PAS 20065, TA is the maximum $\Delta L$ in the entire spectrum. However, it is important to take each component into account to better understand the actual situation, especially in the case of noise with multiple tonal components. Thus, the prototype displays the largest three TAs on the screen as shown in Fig. 2 and outputs files in csv format including all detected TA values. The display updates every three seconds.

\section{Accuracy validation}

The prototype software is validated by the laboratory experiment in an anechoic chamber through the measurement

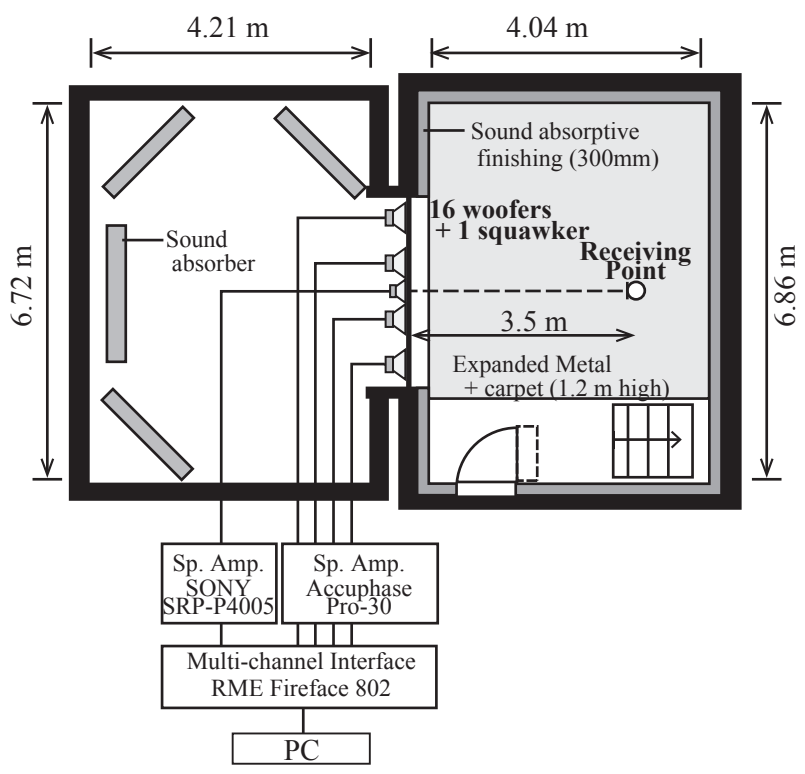

Fig. 3 Arrangement of accuracy validation measurement.

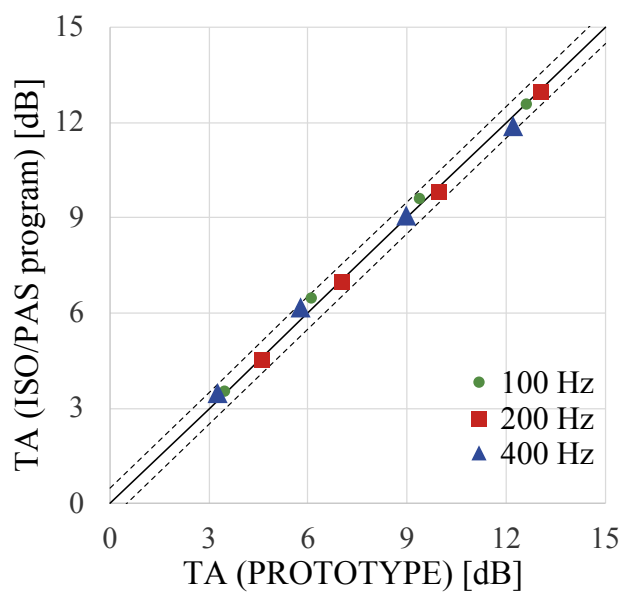

Fig. 4 TA results of artificial signals.

of the test signals with tonal components according to the measurement arrangement shown in Fig. 3. The artificial sound (Sect. 3.1) and measured sound in-situ (Sect. 3.2) are used as test signals. Each test sound is played for 15 seconds, of which three 3 -second data is measured in real time using the prototype software. The energy averaged results are compared with the data from the almost same time that are analyzed later by the attached sample program of ISO/PAS 20065.

3.1. Measurement of artificial tonal noise

The test signals comprise of the broadband noise with a frequency characteristic of $-4 \mathrm{~dB} /$ octave, to which tonal components at 100,200, and $400 \mathrm{~Hz}$ are added, respectively. Each signal is tuned to exhibit TA values of 3, 6, 9, and $12 \mathrm{~dB}$ at the receiving point in Fig. 3, respectively. Figure 4 reveals the results of the prototype software in the horizontal axis and that of ISO/PAS 20065 in the vertical axis. The two results are in good agreement with a difference of less than $0.4 \mathrm{~dB}$. 
Table 1 TA results of measured signals.

\begin{tabular}{|c|c|c|c|c|c|c|c|c|c|c|}
\hline \multirow{2}{*}{$\begin{array}{c}\text { Test } \\
\text { signals }\end{array}$} & \multirow{2}{*}{$\begin{array}{c}L_{\mathrm{A}} \\
(\mathrm{dB})\end{array}$} & \multirow{2}{*}{$\begin{array}{c}\text { Tone } \\
\text { frequency } \\
(\mathrm{Hz})\end{array}$} & \multicolumn{2}{|l|}{$1 \mathrm{st}$} & \multicolumn{2}{|l|}{ 2nd } & \multicolumn{2}{|c|}{$3 \mathrm{rd}$} & \multicolumn{2}{|c|}{ Average } \\
\hline & & & PROTOTYPE & ISO/PAS & PROTOTYPE & ISO/PAS & PROTOTYPE & ISO/PAS & PROTOTYPE & ISO/PAS \\
\hline WTN1 & 51.5 & 165.6 & 10.0 & 10.0 & 8.7 & 8.5 & 8.3 & 8.7 & 9.1 & 9.1 \\
\hline WTN2 & 47.7 & 175.0 & 14.0 & 15.9 & 11.9 & 12.0 & 13.8 & 12.7 & 13.4 & 13.9 \\
\hline \multirow{2}{*}{ LWN } & \multirow{2}{*}{54.3} & $1,737.5$ & 20.9 & 21.2 & 21.6 & 20.0 & 21.7 & 20.6 & 21.4 & 20.6 \\
\hline & & $2,440.6$ & 20.7 & 20.6 & 20.8 & 22.5 & 20.6 & 20.9 & 20.7 & 21.4 \\
\hline
\end{tabular}

Therefore, the accuracy of the prototype software is confirmed for the artificial signals.

\subsection{Measurement of actual tonal noise}

The test signals are two wind turbine noise WTN1 and WTN2 measured by Tachibana et al. [9] and one louver wind noise LWN measured by Tomitaka et al. [10] as shown in Table 1. The louver wind noise has many tonal components. Table 1 also shows the A-weighted sound pressure level $L_{\mathrm{A}}$, the measurement data, and the average. The results of the prototype software agree well with that of ISO/PAS sample program with a difference of less than $0.8 \mathrm{~dB}$. Further, it is indicated that the noise containing multiple tonal components like louver wind noise can also be evaluated.

\section{In-situ measurements}

The tonal noise produced from the exhaust port at the building of the hypersonic wind tunnel (Bldg. 2 in Fig. 5) in Kashiwa campus, The University of Tokyo, is measured in real time. The exhaust port is located $3.4 \mathrm{~m}$ higher than the

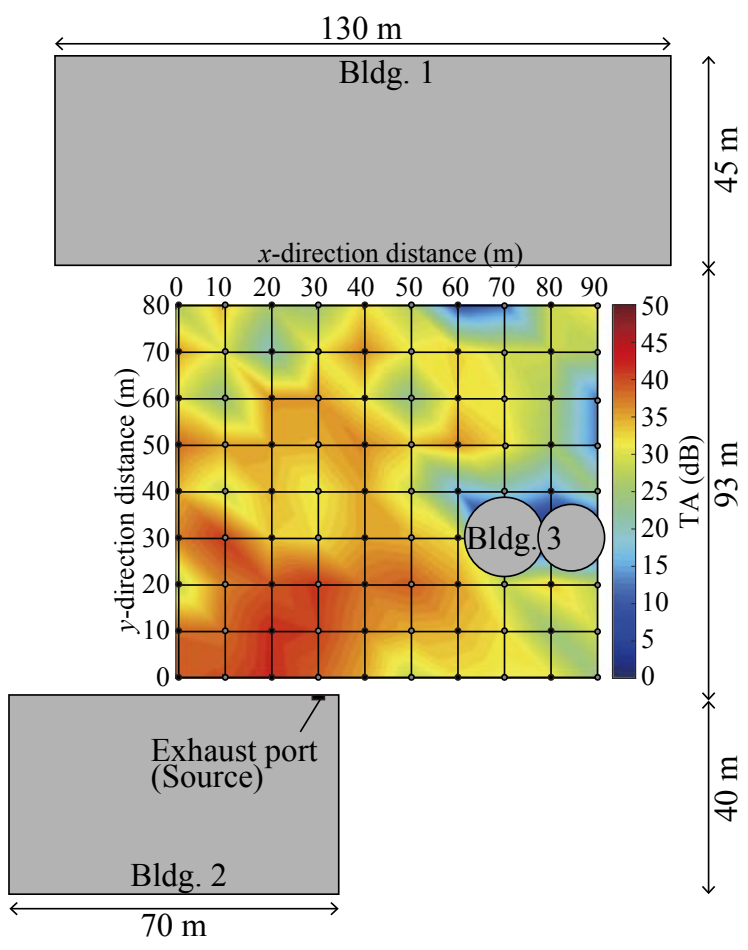

Fig. 5 2D TA distribution. soil ground. From Bldg. 2, there is a large open space (soil) across the street with Bldg. 1 on the opposite side. Additionally, the conical building, Bldg. 3, also exists at the east side of the open place. The TA of the entire field in front of the Bldg. 2 (4.1 2D measurement) is first measured. The TA in line with the front of the outlet (4.2 1D measurement) is obtained after to measure the more detailed distribution.

\subsection{D measurement}

A TA distribution of $10 \times 9$ matrix $(90 \mathrm{~m} \times 80 \mathrm{~m})$ is measured every $10 \mathrm{~m}$ as shown in Fig. 5. Three 3-second measurements are carried out at each receiving point and the results are their energy averaged data. TA distribution of $130 \mathrm{~Hz}$, which is the most prominent tone, is shown in Fig. 5. Although some influence of the interference is evident, TA generally decreases with distance from the sound source. In addition, TA values are low especially at the back side of Bldg. 3.

4.2. 1D measurement

TA distribution in line with the front of the outlet is measured every $2.5 \mathrm{~m}$. Three 3 -second measurements are carried out at each receiving point, and the results are their energy averaged data. TA distribution of $130 \mathrm{~Hz}$ calculated by the prototype software is shown in Fig. 6. A-weighted sound pressure level $L_{\mathrm{A}}$ and that of $130 \mathrm{~Hz}$ (hereinafter, $L_{\mathrm{A}, 130 \mathrm{~Hz}}$ ) obtained by FFT function of the device are also plotted in the same figure.

According to TA data, dips appear at $5 \mathrm{~m}, 30 \mathrm{~m}$ points, etc. $L_{\mathrm{A}, 130 \mathrm{~Hz}}$ result also exhibits similar dips but $L_{\mathrm{A}}$ does not. One possible cause is that the interference of the direct sound and that of the reflected decreases the sound pressure of $130 \mathrm{~Hz}$. To confirm this, a simple geometric calculation is

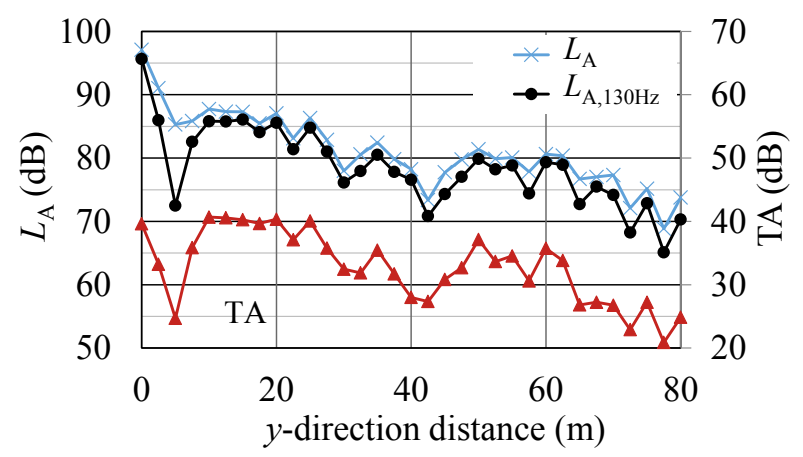

Fig. 6 1D TA distribution. 


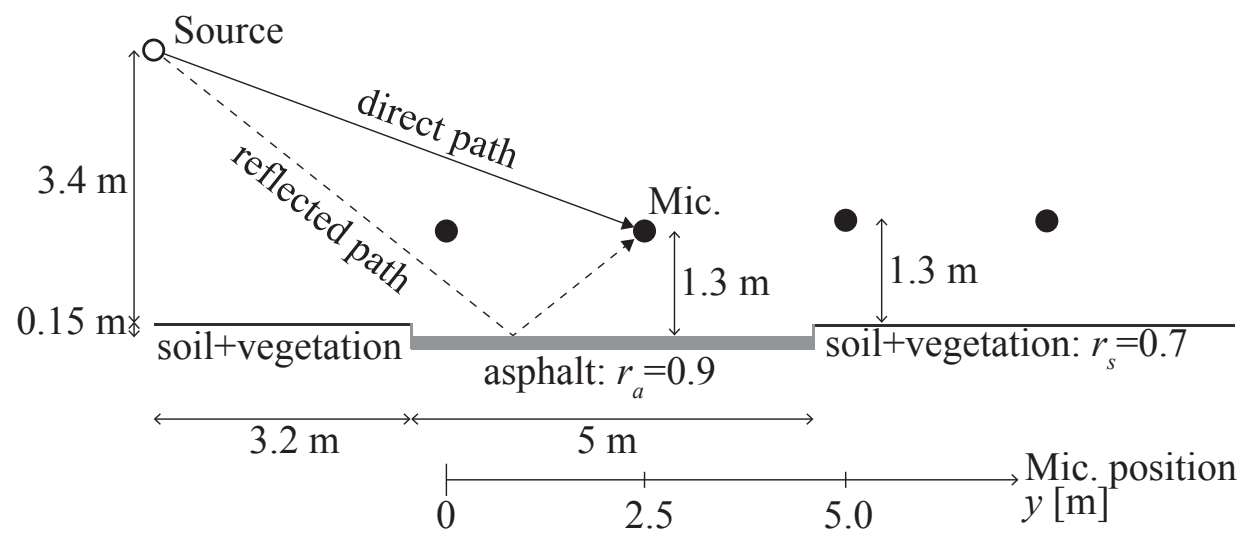

Fig. 7 Geometrical settings for calculation.

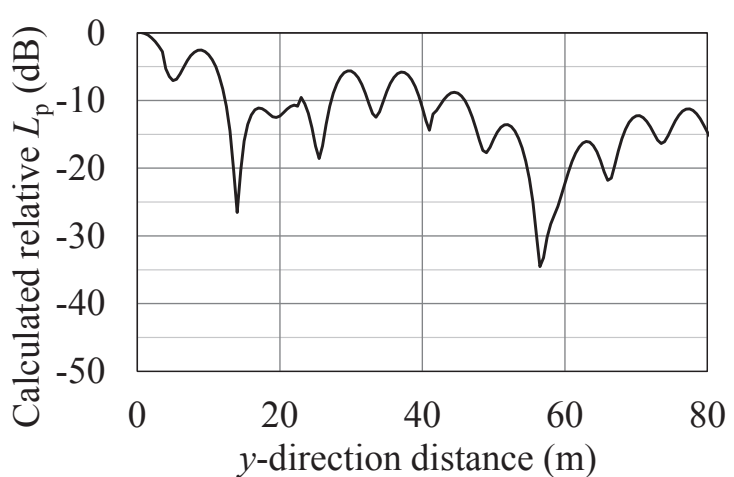

Fig. 8 Calculation result of RSPL distribution of $130 \mathrm{~Hz}$.

carried out. The superposition of the direct and reflected sound waves when the sound wave of $130 \mathrm{~Hz}$ is radiated from the sound source point is calculated according to the geometrical settings shown in Fig. 7. The reflection coefficients of the asphalt and the soil are set to 0.9 and 0.7 , respectively. The geometrical calculation is carried out every $0.1 \mathrm{~m}$ from $y=0 \mathrm{~m}$ to $y=80 \mathrm{~m}$. The obtained relative sound pressure levels (RSPL) normalized by that of the source point are plotted in Fig. 8. According to the results, the dips occur at positions similar to the measurement data, which are then concluded to be the influence of the interference.

\section{Conclusion}

A measuring application that works with a tablet device with AD conversion and FFT function is created for tonal audibility measurement. From the results, the accuracy of the prototype software is validated through the measurement in anechoic chamber. In addition, the findings in this letter suggest that this system can be a practical tool that enables simple real-time measurements.

\section{Acknowledgement}

The authors are grateful to Tomohiro Kobayashi (Kobayasi Institute of Physical Research) for technical advices on the program development. This study was supported by the Environment Research and Technology Development Fund JPMEERF20175010 of the Environmental Restoration and Conservation Agency of Japan.

\section{References}

[1] ISO 1996-2:2017 Acoustics - Description, measurement and assessment of environmental noise - Part 2: Determination of sound pressure levels (2017).

[2] ISO/PAS 20065:2016 Acoustics - Objective method for assessing the audibility of tones in noise-Engineering method (2016).

[3] IEC 61400-11:2012 Wind turbines - Part 11: Acoustic noise measurement techniques (2012).

[4] H. Fletcher, "Auditory patterns," Rev. Mod. Phys., 12(47), 4765 (1940).

[5] B. C. J. Moore, An Introduction to the Psychology of Hearing, 6th ed. (Brill, Leiden, 2013).

[6] H. Tachibana, "Guidelines for wind turbine noise in foreign countries," J. Acoust. Soc. Jpn. (J), 71, 198-205 (2015) (in Japanese).

[7] S. Yokoyama, T. Kobayashi and H. Tachibana, "Perception of tonal components contained in wind turbine noise," Proc. Internoise 2016, Hamburg, pp. 2628-2639 (2016).

[8] M. Yonemura, H. Lee and S. Sakamoto, "Study on subjective evaluation of noise containing low frequency tonal components assuming time of sleeping," Proc. Autumn Meet. Acoust. Soc. Jpn., pp. 433-436 (2019) (in Japanese).

[9] H. Tachibana, H. Yano, A. Fukushima and S. Sueoka, "Nationwide field measurements of wind turbine noise in Japan," Noise Control Eng. J., 62, 90-101 (2014).

[10] T. Tomitaka, A. Tabata and Y. Yoshikawa, "Investigation on the evaluation of aerodynamic noise emitted from buildings claddings - The present conditions and the problem -," Tech. Rep. Archit. Acoust. Acoust. Soc. Jpn., AA2010-5, pp. 1-8 (2010) (in Japanese). 